\title{
Attention Analysis and Information Dissemination Effect of Young People Based on Multisource Sensors under the Background of Wireless Communication and Artificial Intelligence
}

\author{
Zheng Liu (iD) ${ }^{1,2}$ and Rong Li (iD) ${ }^{2}$ \\ ${ }^{1}$ School of Journalism, Fudan University, Shanghai 200433, China \\ ${ }^{2}$ School Humanities \& Communication, Zhejiang Gongshang University, Hangzhou 310002, China \\ Correspondence should be addressed to Rong Li; lirong_vip@outlook.com
}

Received 19 November 2021; Revised 11 January 2022; Accepted 12 January 2022; Published 3 March 2022

Academic Editor: M Pallikonda Rajasekaran

Copyright (c) 2022 Zheng Liu and Rong Li. This is an open access article distributed under the Creative Commons Attribution License, which permits unrestricted use, distribution, and reproduction in any medium, provided the original work is properly cited.

\begin{abstract}
In recent years, the development of wireless communication and artificial intelligence technology has brought tremendous convenience to human life. Especially in the fields of education and medical treatment, the functions of wireless communication are the most representative. In order to enhance the attention of young people and the effect of information dissemination, scholars have conducted many analyses on its influencing factors. Studies have found that strong noise environments have obvious negative effects on attention and information dissemination effects. Therefore, this paper proposes a multisource sensorbased method for young people's attention analysis and information dissemination effect enhancement in a strong noise environment. Multisource sensors are selected to perform young people's attention analysis on speech in a strong noise environment, combined with the information dissemination effect to strengthen the model obtained by combining attention analysis and information dissemination effect strengthened speech. Through the multisource sensor system, it is demonstrated that the method in this paper is superior to the traditional method in a strong noise environment. And through the comparative analysis of multisource sensors, the corresponding improvement plan for the strong noise environment was formulated, and the numerical simulation experiment of young people's attention analysis and information dissemination effect was carried out to verify the optimization results. This research, based on wireless communication and artificial intelligence decision-making technology, proposes to evaluate and optimize the attention analysis and information dissemination effect of young people in a strong noisy environment based on multisource sensors and gives useful ideas to ensure the system design method in a strong noisy environment and the environmental quality after implementation. It also provides support and help for people to more specifically prevent the adverse effects on people's attention analysis and information dissemination effects in a strong noise environment. This paper designs a model based on multisource sensors to study the effect of young people's attention analysis and information dissemination in a strong noise environment, which is of great significance to the development of people's high-quality life.
\end{abstract}

\section{Introduction}

Strong noise environment refers to the sound that hinders people's attention and transmission effect in a space surrounded by materials and separated from the outside. Since the industrial revolution, the environment with strong noise has been a key source of human physical and psychological stress and has caused troubles to a large number of young people who must perform key tasks in the environment with strong noise [1]. Until the rapid development of information technology, especially wireless communication and artificial intelligence decision-making technology, this problem has not been effectively solved for a long time. Since the 1970s, scholars have conducted many studies on the effect of strong noise environment on 
attention analysis and information dissemination, and most of the experimental results also show that strong noise environment has obvious adverse effects on attention and information dissemination effects [2]. Strong noise environment is often a key source of human physical and mental stress. In modern society, because people spend about $80 \%$ to $90 \%$ of their lives indoors, and the auditory stimuli produced by handheld devices through voice and music are everywhere, it even covers other senses and the duration of noise in a strong noise environment is more than usual [3]. Therefore, analyzing how a strong noise environment affects attention and the effect of information dissemination is critical to enriching the relevant literature and preventing noise [4]. In the previous analysis on the strong noise environment, many studies have been carried out on the connection between the strong noise environment and the attention analysis, but the research on the influence of the attention analysis and the information dissemination effect of young people in the strong noise environment is not comprehensive [5]. In addition, existing research shows that the impact of forced noise environment on young people's attention analysis and transmission effect is determined to a certain extent based on the evaluation level of multisource sensors [6].

The multisource sensor is not sensitive to the noise transmitted by the air, so it has a strong anti-interference function [7]. In the noise situation, the superiority of the sensor system based on wireless communication and artificial intelligence decision-making technology is very obvious; the frequency spectrum of the multisource sensor is very clear and is generally not affected by the strong noise environment [8]. According to this feature, in the strong noise environment, through making full use of the foundation of wireless communication technology, we can construct a sensor system to assist teaching, the attention of young people can be analyzed, and the effect of information dissemination can be evaluated based on the multisource sensor [9]. Research on the combination of multiple sensors and artificial intelligence technology has also received a lot of attention from scholars in the past two years. Therefore, this article uses multisource sensor technology as the basis to study the attention analysis and information dissemination effect of young people in a strong noisy environment and build an evaluation system based on multisource sensors. Strong noise environment has a major impact on work and young people's attention [6, 10-12]. Based on multisource sensors, it further explores and demonstrates the influence of noise on young people's attention and the effect of information dissemination in a strong noise environment, which is an extension of research results in many fields [13].

\section{Materials and Methods}

This research is based on wireless communication and artificial intelligence decision-making technology. The method selection and design are all based on this foundation.

2.1. Attention Test Content. Stroop effect refers to the interference effect of word meaning on font color. It is generally believed that reading a word and speaking the color of a font are two different cognitive processes. Use the Stroop character and color intervention experiment commonly used in psychology to measure the attention of the testees. Provide a text that expresses color, but this text is supplemented with nonliteral colors, allowing the testee to distinguish the content of the font or the meaning of the word [14]. There are ten sets of questions, and the measurement system separately registers the correct number, correct time, wrong number, and wrong time [15-18]. Attention score is "number of correct answers/total time to answer."

2.2. Statistical Analysis Method of the Noise Signal. In order to test the results under different noise conditions, design comparative experiments. First, in order to compare the influence of various noise standards on work efficiency, 8 noise standards are set, they are (a) $50 \mathrm{~dB}$ continuous meaningful noise; (b) $50 \mathrm{~dB}$ continuous non-meaningful noise; (c) $50 \mathrm{~dB}$ intermittent meaningful noise; (d) $50 \mathrm{~dB}$ intermittent meaningless noise; (e) $70 \mathrm{~dB}$ continuous meaningful noise; (f) $70 \mathrm{~dB}$ continuous meaningless noise; (g) $70 \mathrm{~dB}$ intermittent meaningful noise; and (h) $70 \mathrm{~dB}$ intermittent meaningless noise. Furthermore, in order to compare the difference between the work efficiency of each noise standard and the quiet state, the corresponding sample $t$ is used to experimentally study all the noise standards and the baseline standard (quiet state).

2.3. Noise Signal Separation and Processing Model. Use a number of mutually independent source signals $S$ to pass through the mixing matrix $A$ to fuse to obtain the monitoring signal $X$, which is expressed as a matrix:

$$
X(t)=A S(t)
$$

If the signal obtained by the system contains environmental noise $n(t)$, if $n(t)$ is an additive monitoring noise vector, then the monitoring signal $X(t)$ is as follows:

$$
X(t)=A S(t)+n(t)
$$

In the situation where neither $S$ nor $A$ is known, the key of the ICA calculation method is to calculate the unmixing matrix $W$ so that the final output signal $Y(t)$ is best close to the source signal $S$ according to a specific criterion, which can be expressed as

$$
Y(t)=W X(t)
$$

At this time, expect both sides of (1):

$$
E(X)=E(A S)=A E(S)
$$

Then, there are

$$
E(S)=A^{-1} E(X)
$$

Usually after centralization, the next step is to perform whitening filtering on the monitoring information $X$. The whitened signal is as follows: 


$$
\widehat{X}=P X
$$

According to the above formula, let the global hybrid array $Q=P A$, which becomes a new monitoring signal, at this time:

$$
\left\{\begin{array}{l}
E\left(\widehat{X} \hat{X}^{T}\right)=E\left(P X X^{T} P^{T}\right)=E\left(Q S S^{T} Q^{T}\right) \\
=Q E\left(S S^{T}\right) Q^{T}=Q Q^{T}=I \\
T=E\left(\widehat{X} \widehat{X}^{T}\right)
\end{array}\right.
$$

Super-Gaussian dispersion and sub-Gaussian dispersion have positive and negative kurtosis values, and the higher the absolute index of kurtosis, the stronger the non-Gaussianness. In the past, the sound signals in human daily life are consistent with the super-Gaussian dispersion. Figure 1 is the probability distribution of super-Gaussian, Gaussian, and sub-Gaussian signals.

It can be seen from Figure 1 that the random process of the super-Gaussian distribution has a wider distribution area and presents a wider tail. The reason why humans' past sound signals conform to the super-Gaussian signal distribution is because the sound signals also have a wider distribution area.

\section{Results}

3.1. Simulation Experiment of Noisy Speech Enhancement in Multisource Sensors. In this paper, the signal-to-noise ratio is selected to conduct an objective evaluation of speech quality. The signal-to-noise ratio is usually a common way to reflect the speech enhancement calculation method for broadband noise distortion. If the noisy speech is expressed by $x(i)$, the pure speech is expressed by $s(i)$, and the enhanced speech is expressed by $s^{\prime}(i)$, the signal-to-noise ratio of the classic style can be expressed as

$$
S N R=10 \log _{10} \frac{\sum_{i} s^{2}(i)}{\sum_{i}\left[s(i)-s^{\prime}(i)\right]^{2}}
$$

The classic signal-to-noise ratio only provides an approximate signal-to-noise ratio. Since the signal changes in real time, the energy of the noise is evenly distributed. Therefore, the signal-to-noise ratio at different times is different. The signal-to-noise ratio allows it to more accurately express the human ear's perception of speech, and the segmented signal-to-noise ratio can be selected, which is defined as follows:

$$
\text { SEGSNR }-\frac{1}{L} \sum_{k=1}^{L} 10 \log _{10}\left[\frac{\sum_{i=m_{k}}^{m_{k}+N-1} 2(i)}{\sum_{i=m_{k}}\left[s(i)-s^{\prime}(i)\right]^{2}}\right]
$$

When the voice signal-to-noise ratio of the noisy microphone is $10 \mathrm{~dB}, 5 \mathrm{~dB}, 0 \mathrm{~dB},-5 \mathrm{~dB}$, and $-10 \mathrm{~dB}$, the voices of 4 speakers are used to simulate according to the procedure described before. The individual signal-to-noise ratio and the segmented signal-to-noise ratio are equally divided, and the conclusions are shown in Table 1. It can be seen from Table 1 that the combined speech signal-to-noise ratio and segmented signal-to-noise ratio are higher than those of the microphone voice only through spectral subtraction and enhancement, and most of the combined speech signal-to-noise ratios are in the throat microphone, above the voice signal-to-noise ratio. The combined algorithm given in this article can enhance the signal-tonoise ratio and segmented signal-to-noise ratio of speech, but when the spectral subtraction method is selected to implement microphone speech enhancement to combine the voice of the larynx microphone, when the signal-tonoise ratio is - 10 decibels, at that time, the voice signal-tonoise ratio of the throat microphone combined with the voice comparison did not increase significantly. In the face of this problem, it is possible to perform in-depth analysis of the weight function.

3.2. Attention Task and Information Dissemination Effect Test Results. Table 2 shows the descriptive statistical conclusions of different noise strengths, noise time forms, and meaningless graphics recognition tasks under different types of noise.

Table 3 shows the descriptive statistical conclusions of the Stroop word-color interference tasks in different noise strengths, noise time forms, and noise types.

Table 4 shows the descriptive statistical results of typing and spelling tasks in different noise strengths, noise time forms, and noise types.

Table 5 shows the descriptive statistical conclusions of different motivation standards, K-L-T tasks, meaningless graphic recognition tasks, and Stroop character color interference tasks in indoor sound conditions.

The research conclusion of repeated measurement of variance shows that the main effect of noise time form is obvious, expressed as intermittent noise action time is longer than continuous noise. The edge of the interactive response of the noise intensity and the time form of the noise is obvious. After the test conclusion shows that, at 70 decibels, the duration of the intermittent noise is higher than that of the continuous noise.

The interactive response edge of noise time form and noise type is obvious. After the test conclusion shows that during meaningful speech noise, the duration of intermittent noise is higher than that of continuous noise; during intermittent noise, the duration of meaningful speech noise is longer than that of meaningless speech. Other main effects and interactive reactions are not obvious. Conclusion shows that there is no significant difference between the noise standard and the baseline standard.

\subsection{Evaluation of the Impact of Different Noise Environments} on the Physiology and Psychology of Young People. Table 6 shows the noise-diastolic blood pressure grouping data. 


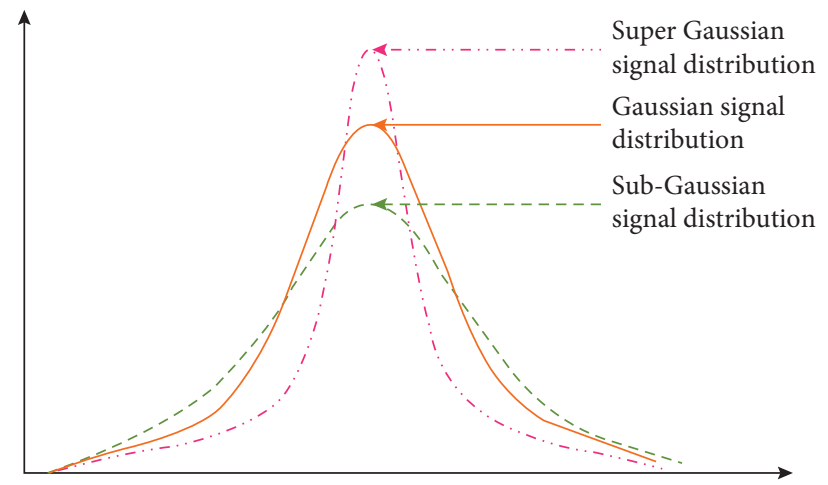

Figure 1: Probability distribution diagram of super-Gaussian, Gaussian, and sub-Gaussian signals.

TABLE 1: The average value of speech signal-to-noise ratio and segmented signal-to-noise ratio when spectral subtraction is used.

\begin{tabular}{|c|c|c|c|c|c|}
\hline \multirow{2}{*}{$\begin{array}{l}\text {-- } \\
\text { Voice with noisy microphone }\end{array}$} & \multicolumn{5}{|c|}{ SNR/segment SNR $(\mathrm{dB})$} \\
\hline & $-10 /-17.34$ & $-5 /-12.33$ & $0 /-7.35$ & $5 /-2.34$ & $10 / 2.66$ \\
\hline Throat microphone voice & & & $79 /-2.82$ & & \\
\hline Microphone voice after spectral subtraction enhancement & $-5.67 /-12.81$ & $-1.02 /-8.1$ & $3.79 /-3.2$ & $8.45 / 1.61$ & $12.86 / 6.3$ \\
\hline Fusion voice & $-0.81 /-7.8$ & $3.69 /-3.22$ & $8.12 / 1.27$ & $12.25 / 5.5$ & $15.91 / 9.4$ \\
\hline
\end{tabular}

TABle 2: Descriptive statistical results of meaningless graphic recognition tasks under different noise conditions (unit: ms).

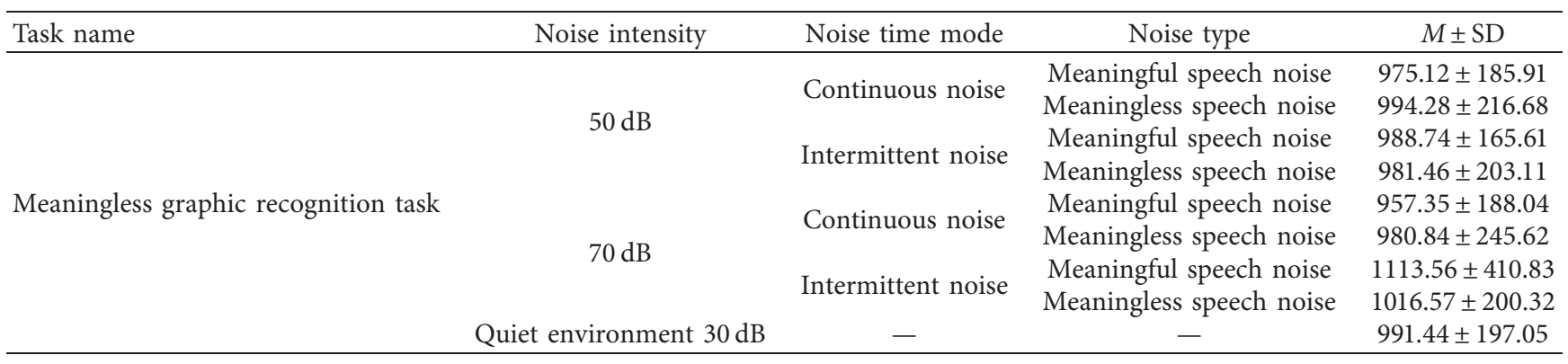

TABLE 3: Descriptive statistical results of Stroop word-color interference tasks under different noise conditions (unit: ms).

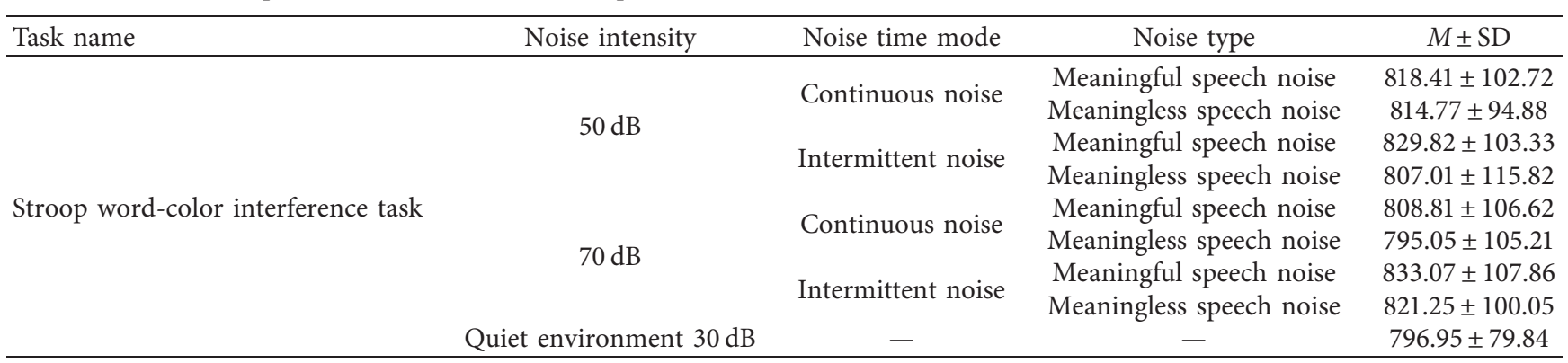

TABLE 4: Descriptive statistical results of typing and spelling tasks under different noise conditions (unit: ms).

\begin{tabular}{|c|c|c|c|c|}
\hline Task name & Noise intensity & Noise time mode & Noise type & $M \pm \mathrm{SD}$ \\
\hline \multirow{9}{*}{ Typing and spelling tasks } & \multirow{4}{*}{$50 \mathrm{~dB}$} & \multirow{2}{*}{ Continuous noise } & Meaningful speech noise & $2963.63 \pm 598.05$ \\
\hline & & & Meaningless speech noise & $2928.48 \pm 643.57$ \\
\hline & & \multirow{2}{*}{ Intermittent noise } & Meaningful speech noise & $2924.81 \pm 608.36$ \\
\hline & & & Meaningless speech noise & $2988.51 \pm 685.65$ \\
\hline & \multirow{4}{*}{$70 \mathrm{~dB}$} & \multirow{2}{*}{ Continuous noise } & Meaningful speech noise & $2979.75 \pm 741.68$ \\
\hline & & & Meaningless speech noise & $2887.25 \pm 635.50$ \\
\hline & & \multirow{2}{*}{ Intermittent noise } & Meaningful speech noise & $2822.62 \pm 647.32$ \\
\hline & & & Meaningless speech noise & $2888.62 \pm 604.68$ \\
\hline & Quiet environment $30 \mathrm{~dB}$ & - & - & $2878.48 \pm 542.82$ \\
\hline
\end{tabular}


Table 5: Descriptive statistical results of the accuracy of each task under different motivation levels and indoor sound environments.

\begin{tabular}{|c|c|c|c|}
\hline Task name & Motivation level & Indoor sound environment & $M \pm \mathrm{SD}$ \\
\hline \multirow{2}{*}{ K-L-T mission } & Low motivation level & $\begin{array}{l}30 \mathrm{~dB} \text { quiet environment } \\
70 \mathrm{~dB} \text { intermittent meaningful noise environment }\end{array}$ & $\begin{array}{l}0.87 \pm 0.06 \\
0.86 \pm 0.07\end{array}$ \\
\hline & High motivation level & $\begin{array}{l}30 \mathrm{~dB} \text { quiet environment } \\
70 \mathrm{~dB} \text { intermittent meaningful noise environment }\end{array}$ & $\begin{array}{l}0.91 \pm 0.03 \\
0.88 \pm 0.03\end{array}$ \\
\hline \multirow{2}{*}{ Meaningless graphic recognition task } & Low motivation level & $\begin{array}{l}30 \mathrm{~dB} \text { quiet environment } \\
70 \mathrm{~dB} \text { intermittent meaningful noise environment }\end{array}$ & $\begin{array}{l}0.92 \pm 0.04 \\
0.91 \pm 0.06\end{array}$ \\
\hline & High motivation level & $\begin{array}{l}30 \mathrm{~dB} \text { quiet environment } \\
70 \mathrm{~dB} \text { intermittent meaningful noise environment }\end{array}$ & $\begin{array}{l}0.92 \pm 0.04 \\
0.91 \pm 0.04\end{array}$ \\
\hline \multirow{2}{*}{ Stroop word-color interference task } & Low motivation level & $\begin{array}{l}30 \mathrm{~dB} \text { quiet environment } \\
70 \mathrm{~dB} \text { intermittent meaningful noise environment }\end{array}$ & $\begin{array}{l}0.91 \pm 0.06 \\
0.93 \pm 0.06\end{array}$ \\
\hline & High motivation level & $\begin{array}{l}30 \mathrm{~dB} \text { quiet environment } \\
70 \mathrm{~dB} \text { intermittent meaningful noise environment }\end{array}$ & $\begin{array}{l}0.87 \pm 0.13 \\
0.88 \pm 0.11\end{array}$ \\
\hline
\end{tabular}

TABLE 6: Noise-diastolic blood pressure group information.

\begin{tabular}{|c|c|c|c|c|c|c|c|c|}
\hline \multirow[t]{2}{*}{ Group } & \multirow[t]{2}{*}{ Number of cases } & \multirow[t]{2}{*}{ Average } & \multirow[t]{2}{*}{ Standard deviation } & \multirow[t]{2}{*}{ Standard error } & \multicolumn{2}{|c|}{$\begin{array}{l}95 \% \text { confidence interval of } \\
\text { the mean }\end{array}$} & \multirow[t]{2}{*}{ Minimum } & \multirow[t]{2}{*}{ Max } \\
\hline & & & & & Lower limit & Upper limit & & \\
\hline 1 & 15 & 74.88 & 7.146 & 1.844 & 71.44 & 82.36 & 58 & 96 \\
\hline 2 & 15 & 72.81 & 8.704 & 2.247 & 67.97 & 77.61 & 57 & 92 \\
\hline 3 & 60 & 75.12 & 8.192 & 1.057 & 73.01 & 77.24 & 53 & 90 \\
\hline 4 & 60 & 73.04 & 7.238 & 0.934 & 71.17 & 74.91 & 57 & 92 \\
\hline 5 & 150 & 74.33 & 8.752 & 0.714 & 72.92 & 75.74 & 51 & 96 \\
\hline
\end{tabular}

Comparing the group data of diastolic and systolic blood pressure, combining Table 7, and comparing the blood pressure of the subjects at rest, the average index of diastolic blood pressure clustering 5 groups and the average resting index from low to high are $2<4<$ rest $<5<1<3$; the average index of the 5 groups of systolic blood pressure and the average resting index from low to high are $2<1<3<5<4<$ rest.

The third and fourth levels of diastolic blood pressure are all rising. The third level includes outdoor speech with a relatively high sound pressure level and car driving sounds with a relatively low sound pressure level. The diastolic pressure of the car driving sound is obviously above the 40decibel floor pedaling sound of the fourth level. The conclusion shows that the third level of noise has a key impact on the increase of diastolic blood pressure, and the fourth level of noise has a more obvious effect on systolic blood pressure.

The fourth level only contains 37 decibels of working noise, and its sound pressure level is not high. The annoying degree of 37 decibels and 55 decibels of work noise is obviously in the same sound pressure level. Above, the working noise of high sound pressure level is at the fifth level, so it can be known that the subjects are more annoyed by this type of noise.

\section{Discussion}

4.1. Correlation Analysis of Strong Noise Environment and Young People's Work Attitude. Based on the above description, this article has formulated five mediation paths for the effect of noise on safety attitudes from the two levels of young people's physical condition (poor physical fitness) and psychological condition (attention, subjective fatigue, and risk preference). Because the age range of the testees used is relatively high-young and middle-aged and old, age is regarded as a control variable.

According to the conclusion of the information, there are 4 paths with prominent mediation effect, these 4 paths are 1-5 except 2; the attention is not prominent and cannot be used as a mediation variable. The index and standard error of each path are shown in Table 8 .

Table 9 shows some mediating effect indexes. It can be seen from the table that "noise $\longrightarrow$ poor physical fitness $\longrightarrow$ safety attitude" is the most critical mediation path, and some of the mediating effect indexes account for the highest proportion of the overall mediating effect index. It is $43.55 \%$. The second is "noise $\longrightarrow$ subjective fatigue $\longrightarrow$ safety attitude."

4.2. The Impact of Strong Noise Environment on Young People's Attention and Attitude. Working in a strong noisy environment, the more physical energy is reduced. This is because, in order to maintain the same production level, the physical strength of young people working in a strong noisy environment is usually higher than that in a noisy environment. This is consistent with the results of previous studies. Based on this, this article further expands and studies poor physical fitness as a key intermediary variable. The conclusion shows that poor physical fitness has an intermediary role in the correlation between noise and safety attitudes. The more physical fitness decreases, the worse the safety attitude becomes. According to the resource theory, 
TABLE 7: Noise-systolic blood pressure grouping information.

\begin{tabular}{lccccccc}
\hline Group & Number of cases & Average & Standard deviation & Standard error & \multicolumn{2}{c}{$\begin{array}{c}\text { 95\% confidence } \\
\text { interval of the mean } \\
\text { Upper limit }\end{array}$} \\
\hline 1 & & & & 1.604 & 104.60 & 111.02 & 85 \\
2 & 60 & 107.81 & 12.434 & 1.551 & 104.69 & 110.89 & 85 \\
3 & 60 & 107.79 & 12.018 & 3.108 & 100.85 & 113.57 & 86 \\
4 & 15 & 108.66 & 12.03 & 2.335 & 104.37 & 113.92 & 80 \\
5 & 30 & 109.15 & 12.794 & 1.114 & 106.88 & 111.29 & 79 \\
\hline
\end{tabular}

TABLE 8: Mediation effect path coefficient.

\begin{tabular}{lcr}
\hline Action path & Path coefficient & SE \\
\hline Noise $\longrightarrow$ safety attitude & -9.5322 & 3.1240 \\
Noise $\longrightarrow$ poor physical fitness & 3.8928 & 1.2935 \\
Poor physical fitness $\longrightarrow$ safe attitude & -0.6791 & 0.2611 \\
Noise $\longrightarrow$ subjective fatigue & -3.6348 & 1.6620 \\
Subjective fatigue $\longrightarrow$ safety attitude & 0.5597 & 0.1405 \\
Noise $\longrightarrow$ risk appetite & -1.996 & 0.8891 \\
Risk Appetite $\longrightarrow$ safety attitude & 0.3227 & 0.1254 \\
Risk appetite $\longrightarrow$ subjective fatigue & 0.6681 & 0.1607 \\
\hline
\end{tabular}

TABLE 9: Individual mediating effect values.

\begin{tabular}{lcc}
\hline Mediating variable influence path & Normalized effect size & Proportion (\%) \\
\hline Noise $\longrightarrow$ poor physical fitness $\longrightarrow$ safety attitude & -2.6442 & 43.55 \\
Noise $\longrightarrow$ subjective fatigue $\longrightarrow$ safety attitude & -2.0347 & 33.51 \\
Noise $\longrightarrow$ risk appetite $\longrightarrow$ safety attitude & -0.6445 & 10.61 \\
Noise $\longrightarrow$ risk appetite $\longrightarrow$ subjective fatigue $\longrightarrow$ safety attitude & -0.7467 & 12.29 \\
Overall mediation effect & -6.0702 & - \\
\hline
\end{tabular}

when the total amount of resources (physical energy) is reduced and the task requirements are not met, it will have a bad impact on work efficiency and risk perception ability. The greater the degree of physical exertion is, the less accurate the perception of the corresponding position and the output power required to achieve a certain posture is, and the more the errors will occur, which will have a negative impact on safety attitudes. Therefore, the company should pay attention to the decline in physical fitness of young people in safety supervision [19]. Moreover, temperature has a regulating effect in the correlation between poor physical fitness and safety attitudes, and temperature reduces the negative effect of poor fitness on safety attitudes [20]. The test conclusion shows that the mediating effect of poor physical fitness at $25^{\circ} \mathrm{C}$ is more pronounced than at $35^{\circ} \mathrm{C}$. This is because the rise in temperature increases the individual's subjective perception of the same physical exertion, and further discomfort creates individual alertness [21]. Therefore, rising temperatures have reduced the degree of negative effects of poor physical fitness on safety attitudes, and the singular cannot reverse this negative effect [22].

The test information shows that noise has a positive effect on attention. The previous analysis table shows that noise is not only at the level of damage. To a certain extent, short-term auditory stimulation can have a positive effect on attention indicators, but long-term noise stimulation has a negative effect on attention [23]. Noise in a specific range as a noxious stimulus can stimulate the limbic system of the subject's brain according to two methods and cause the subject's attention to produce a stress effect [24]. One method is that noise stimulates the thalamic adrenal axis to produce a large amount of adrenaline and adrenaline cortex, which can process more blood sugar and supply a lot of energy for voluntary muscles and sensory [25]. The second method is that noise stimulates sympathetic nerves and vagus nerves. Let the involuntary muscles in the heart and lungs produce a lot of blood, and the brain can get more oxygen. Moreover, air temperature can adjust the effect of noise on attention. When the air temperature rises, it will reduce the positive effect of noise on attention and turn the effect of noise on attention into a negative one. Because when a stimulus element is generated in the environment, the subject will experience a stress response in a short period of time, the central nervous system will be excited, and the adrenaline will rise, which positively acts on the subject's attention, and the subject is more alert; but in the environment when a large number of stimulating elements are produced together, the subject is in a poor working environment, with a sharp decline in physical and psychological conditions and a decrease in concentration [26]. In addition, the test information shows that the mediating role of attention is not prominent. The reason may be that the 
attention index is obviously related to safety behavior. The lack of attention easily leads to the neglect of safe operation process and the error of production behavior. The key to safety attitude reflects behavioral intentions, not a detailed behavior.

\subsection{Research on Work Management Strategies under the} Strong Noise Environment. Because noise in a high-noise working environment is more harmful to safety production, when selecting young people in a high-noise working environment, young people who are not sensitive to noise should be selected, and those who are not sensitive to noise are interfered by noise. The degree is even smaller. Decreased physical fitness and lower risk appetite indicators in a strong noise environment are the reasons for poor safety attitudes. Therefore, when recruiting such young people, it is necessary to select personnel with better initial physical fitness and careful attention. Educational background can adjust the direct effect of noise on safety attitudes, as well as the effect of subjective fatigue on safety attitudes. Even if academic qualifications reduce the level of the positive effect of subjective fatigue on safety attitudes, it cannot change this positive effect. And academic qualifications reduce the negative effect of noise on safety attitudes. Therefore, the degree of education should not be too low or too high, and junior high school or high school graduates should be selected.

When working in a strong noise environment, young people can be equipped with earplugs to reduce noise from the noise receiving place, thereby improving the young people's work concentration. The weakening of noise reduces the reduction of young people's physical fitness after work and also enhances young people's subjective perception and early warning of physical exertion. The reduction of the subject's risk preference indicators reduces, and the physical and psychological conditions can remain stable, which is useful for improving safety attitudes. Moreover, when young people work in a strong noisy environment, they must take a break and replenish their physical fitness at intervals. The company can distribute energy drinks and food for them to reduce the harm of physical loss to safety attitudes. And when young people are resting, they can stimulate the subjective sense of fatigue that has been interfered by noise before, and the brain safety awareness center will efficiently improve and process this information, so that young people can get scientific safe behavior decisions. If you want to work in a strong noise environment for a long time, the company must design a scientific shift strategy.

\section{Conclusion}

Wireless communication and artificial intelligence technology make the function of the sensor more powerful, and the function it can play is also more comprehensive. The correlation between attention and noise presents a significant negative index. The stronger the noise (strong or weak), the worse the attention. Noise essentially represents redundant annoying sounds. Noise can potentially disturb young people, have a bad effect on young people's psychology and cognition, significantly reduce the level of work efficiency, and conceal the prewarning signals and warnings of unexpected accidents. At the same time, the degree of education reduces the negative effect of noise on attention, because the higher the degree of education, the higher the psychological flexibility of the teste, and the higher the ability of the teste to control, restrict, and regulate themselves and face a poor working state. The adaptability is stronger here, and it can further acquire positive and accurate attitudes, so the negative effect of noise on safety attitudes is reduced. When the effect of attention and information dissemination is high, better perception abilities, emotions, and reaction abilities can have a positive effect on the subject's subjective perception of fatigue and further have a positive effect on attention and information dissemination. Noise reduces the risk appetite index of the test subject, so the subjective perception of physical exertion is also reduced. With the subjective failure to accurately perceive this kind of physical exertion, the level of reflection of the safety awareness center of the brain will decrease, and the young people's attention will not be high.

Based on the evaluation of multisource sensors, this paper conducts research on the noise in the strong noise environment and discusses the current situation of the noise influence in the strong noise environment. The existing analysis on the attention of noise and the effect of information dissemination mostly focuses on the influence of strong noise. The research on the type of noise is relatively monotonous, after long-term observation on the attention of strong noise. This paper carried out the analysis. This paper mainly studies the impact of four types of noise sources on the attention and information dissemination effect of young people in the life level in a strong noise environment and divides the combination of various noise types according to the testee's attention and the coefficient of information dissemination effect. And through analysis, it is found that noise in a strong noise environment has a negative impact on attention and information dissemination. Therefore, the company should pay attention to the improvement of the working environment and improve the attention of young people according to noise reduction methods to ensure safe work. And noise disturbs young people's subjective perception of physical exertion. When the brain fails to accurately obtain fatigue information, the level of attention response decreases and attention is poor. Therefore, the company should plan the rest time at work, which will help young people to activate the attention that was disturbed by noise before, and it will also help to improve their safety attitude.

In the future, we can also combine big data technology to analyze a large amount of empirical data, so as to better prove the authenticity of this article. The advancement of the Internet of Things technology will also make the sensor technology more advanced. In the future, the most cuttingedge sensors can be used, so that high-performance sensors can obtain more accurate sample data, thereby obtaining more accurate experimental data. 


\section{Data Availability}

The datasets used and/or analyzed during the current study are available from the corresponding author upon reasonable request.

\section{Additional Points}

This article is licensed under a Creative Commons Attribution 4.0 International License, which permits use, sharing, adaptation, distribution, and reproduction in any medium or format, as long as you give appropriate credit to the original author(s) and the source, provide a link to the Creative Commons license, and indicate if changes were made. The images or other third-party materials in this article are included in the article's Creative Commons license, unless indicated otherwise in a credit line to the material. If material is not included in the article's Creative Commons license and your intended use is not permitted by statutory regulation or exceeds the permitted use, you will need to obtain permission directly from the copyright holder. To view a copy of this license, visit http:// creativecommons.org/licenses/by/4.0/.

\section{Conflicts of Interest}

The authors declare that they have no conflicts of interest.

\section{Acknowledgments}

This work was supported by the Projects of the Human Social Science on Young Fund of the Ministry of Education, "Research on the Institutional History of French Communication under the New Cultural History Paradigm" (19YJC860031).

\section{References}

[1] A. Ahmed, T. Khan, S. Jahandad, M. H. Hakimi, A. A. Lashin, and N. S. Z. Abidin, "Organic geochemistry indicates sourcerock characteristics and hydrocarbon potential: A case study from Early Cretaceous Sembar Formation, southern Indus Basin, Pakistan," Arabian Journal of Geosciences, vol. 13, no. 23, p. 1234, 2020.

[2] D. Akbarimehr, A. Eslami, E. Aflaki, and R. Imam, "Using empirical correlations and artificial neural network to estimate compressibility of low plasticity clays," Arabian Journal of Geosciences, vol. 13, no. 22, p. 1225, 2020.

[3] S. B. Hu, M. Q. Zhu, L. L. Yang et al., "Future wireless communication and big data, artificial intelligence," Journal of Guizhou Normal University (Natural Science Edition), vol. 38, no. 06, pp. 1-10+132, 2020.

[4] L. L. Ma, J. Guo, S. K. Wang et al., "Multi-source sensor fault diagnosis method based on improved CNN-GRU network," Journal of Beijing Institute of Technology, vol. 41, no. 12, pp. 1245-1252, 2021.

[5] C. Zhao, S. M. Zhong, X. J. Zhang et al., "Novel results on nonfragile sampled-data exponential synchronization for delayed complex dynamical networks," International Journal of Robust and Nonlinear Control, vol. 30, no. 9, pp. 4022-4042, 2020.
[6] J. N. Luo, M. L. Li, X. Z. Liu, W. H. Tian, S. M. Zhong, and K. B. Shi, "Stabilization analysis for fuzzy systems with a switched sampled-data control," Journal of the Franklin Institute, vol. 357, no. 1, pp. 39-58, 2020.

[7] Y. X. Deng, W. Guo, and K. T. Li, "Single satellite positioning algorithm and accuracy analysis based on multi-source sensors," Firepower and Command Control, vol. 46, no. 02, pp. 126-132, 2021.

[8] G. L. Chen and X. X. Cao, "Pedestrian behavior recognition in indoor fire based on mobile phone multi-source sensors," Journal of Tongji University, vol. 47, no. 03, pp. 414-420, 2019.

[9] C. Zhao, S. M. Zhong, Q. S. Zhong, and K. B. Shi, "Synchronization of Markovian complex networks with input mode delay and Markovian directed communication via distributed dynamic event-triggered control," Nonlinear analysis. Hybrid systems, vol. 36, p. 100883, 2020.

[10] F. Liu, G. Zhang, and J. Lu, "Heterogeneous domain adaptation: An unsupervised approach," IEEE Transactions on Neural Networks and Learning Systems, vol. 31, no. 12, pp. 5588-5602, 2020.

[11] F. Meng, W. Cheng, and J. Wang, "Semi-supervised software defect prediction model based on tri-training," Ksii Transactions on Internet and Information Systems, vol. 15, no. 11, pp. 4028-4042, 2021.

[12] T. Sui, D. Marelli, X. Sun, and M. Fu, "Multi-sensor state estimation over lossy channels using coded measurements," Automatica, vol. 111, p. 108561, 2020.

[13] B. C. Chen, "Treatment of attention deficit hyperactivity disorder in children and adolescents-viloxazine hydrochloride (viloxazine hydrochloride) sustained-release capsules," Medical Herald, vol. 40, no. 12, pp. 1785-1793, 2021.

[14] X. L. Yang, "Analysis of potential problem prevention strategies in youth sports teaching," Shang $W u$, vol. 2021, no. 10, pp. 109-110, 2021.

[15] R. D. Luo and Y. D. Guo, "Will the advancement of artificial intelligence technology promote the common prosperity of enterprise employees? [J/OL]," Guangdong Social Sciences, vol. 2022, pp. 1-10, 2022, [2022-01-07].

[16] Z. Wu, C. Li, J. Cao, and Y. Ge, "On scalability of associationrule-based recommendation," ACM Transactions on the Web, vol. 14, no. 3, pp. 1-21, 2020.

[17] Z. Wu, A. Song, J. Cao, J. Luo, and L. Zhang, "Efficiently translating complex SQL query to MapReduce Jobflow on cloud," IEEE transactions on cloud computing, vol. 8, no. 2, pp. 508-517, 2020.

[18] S. Balraj, S. Parveen, P. Abbas, and A. Angelaki, "Comparative analysis of artificial intelligence techniques for the prediction of infiltration process," Geology, Ecology, and Landscapes, vol. 5, no. 2, pp. 109-118, 2021.

[19] G. H. Zhan, T. Liu, and F. Y. Dai, "Research on the integration of intelligent teaching space in colleges and universities driven by artificial intelligence [J/OL]," Journal of Ningbo University (Educational Science Edition), vol. 2022, pp. 1-9, 2022, [202201-07].

[20] Z. Q. Hu and C. J. Zhi, “The development and application of software testing in the era of artificial intelligence," Electronic World, vol. 2021, no. 24, pp. 101-103, 2021.

[21] L. Li, "Optimized design of wireless power transmission system based on information technology," Electronic World, vol. 2021, no. 24, pp. 198-199, 2021.

[22] S. M. Li, "The harm of earphone noise to human hearing and its prevention," Biology Teaching, vol. 44, no. 12, pp. 64-65, 2019. 
[23] Y. R. Meng, The perception and development of the phoneme category of adolescents in Mandarin under speech noise, Central China Normal University, Hubei, China, 2019.

[24] W. L. Wu and Y. Bai, "Investigation and analysis of the influence of entertainment noise on the hearing of adolescents," Heilongjiang Medicine, vol. 29, no. 05, pp. 983-985, 2016.

[25] X. F. Zhu and C. Xu, "Research progress of hearing impairment in adolescents," Chinese Modern Doctor, vol. 53, no. 11, pp. 153-156, 2015.

[26] Q. H. Zhang and C. Zhang, "Birdsong sound detection method of circular array wireless sensors [J/OL]," Applied Acoustics, vol. 2022, pp. 1-8, 2022, [2022-01-07]. 